\title{
Resolution of Laryngeal Oedema in a Patient with Acquired C1-Inhibitor Deficiency. A Case Report
}

\author{
Noémi-Anna Bara ${ }^{1}$, Valentin Nadasan ${ }^{2 *}$ \\ 1 Hereditary Angioedema Expertise Centre, Sangeorgiu de Mures, Romania \\ 2 George Emil Palade University of Medicine, Pharmacy, Science, and Technology of Targu Mures, Romania
}

\begin{abstract}
Introduction: Laryngeal oedema caused by acquired angioedema due to C1-inhibitor deficiency (C1-INH-AAE) is a life-threatening condition. The swelling is bradykinin mediated and will not respond to the usual treatment with antihistamines, corticosteroids, or epinephrine. Instead, kallikrein-bradykinin-targeted therapies should be used promptly to prevent asphyxiation. Case presentation: A 43 years old female presented at the Hereditary Angioedema Centre reporting a one-year history of peripheral, facial, and neck oedema. Treatment with antihistamines and corticosteroids had been ineffective. Laboratory results showed complement level deficiencies and monoclonal gammopathy characterised as immunoglobulin M. An abdominal ultrasound revealed splenomegaly. A bone marrow biopsy was normal. Based on these data, the diagnosis of C1-INH-AAE associated with monoclonal gammopathy of uncertain significance (MGUS) was made. As C1-INH-AAE can present with life-threatening, standard treatment-resistant laryngeal oedema, an emergency care treatment plan was proposed, and the patient was advised to present to the emergency department (ED) with this medical letter. Based on these recommendations, three laryngeal attacks were successfully treated in the ED with recombinant human C1-inhibitor (two attacks) and fresh frozen plasma (one attack). After these episodes, the patient was prescribed prophylactic treatment with antifibrinolytics. No further angioedema attacks were reported by the patient at the 18 months follow-up visit. Conclusions: Because angioedema of the upper airways is a life-threatening condition, recognising the specific type of swelling by the emergency physician is critical in providing immediate and effective treatment to reduce the associated risk of asphyxiation. C1-INH-AAE being a rare disorder, patients should have available an emergency care treatment plan with recommendations of acute treatment possibilities.
\end{abstract}

Keywords: acquired C1-inhibitor deficiency, laryngeal oedema, asphyxiation, monoclonal gammopathy of uncertain significance, emergency care treatment plan

Received: 10 September 2020 / Accepted: 8 January 2021

\section{INTRODUCTION}

Angioedema manifests as a swelling of the skin or mucosa. It is considered a consequence of a sudden local increase in vascular permeability with histamine, bradykinin, and other mediators. The more frequent histamine-mediated angioedema responds well to antihistamines, corticosteroids, or epinephrine. A less common form of angioedema is bradykinin-mediated, drug-induced, acquired, or hereditary $[1,2]$. In these latter forms, kallikrein-bradykinin-targeted therapies should be used [4]. Identifying the triggering mediator of the swelling may be difficult especially in the ED, where failure to discriminate between different types of angioedema may prevent the effective treatment of the less common conditions [2,3].

Acquired angioedema (AAE) due to $\mathrm{C} 1$-inhibitor (C1-INH) deficiency (C1-INH-AAE) is a rare condition estimated to occur in 1:100,000 to $1: 500,000$ individuals. The number of cases previously reported in the literature is less than a thousand [4-6]. The clinical symptoms include unpredictable attacks of cutaneous or mucosal swelling. While oedema may present at any site, the head, including face, tongue, and lips, the neck, including the larynx, and limbs are most frequently affected. Various degrees of anoxic brain injury 
or even death due to upper airway-oedema is observed in about half of the patients with C1-INH-AAE [6-8].

\section{CASE PRESENTATION}

A 43-year woman, in October 2018, attended the Hereditary Angioedema Centre, reporting a one-year history of recurrent angioedema without urticaria. Symptoms in her hands and legs had started when she was of forty-two years old. Oedema usually lasted one to two days. She reported having a facial and external-neck swelling episode and colicky abdominal pain episodes with nausea and vomiting, after tooth extraction. Two days after a cholecystectomy she developed massive tongue oedema. Treatment with antihistamines and corticosteroids was ineffective.

Allegedly, the patient was given neither angiotensinconverting enzyme inhibitor nor angiotensin receptor blocking medication. She had no family history of angioedema and denied having any drug or food allergies.

Laboratory investigations, at the time of admission to the Hereditary Angioedema Centre, revealed complement deficiencies with low C4 level $(2.9 \mathrm{mg} / \mathrm{dL}$; normal values $15-57 \mathrm{mg} / \mathrm{dL}$ ), low $\mathrm{C} 1-\mathrm{INH}$ antigenic level (4.3 mg/dL; normal values 21-39 mg/dL), low C1-INH activity (12\%; normal values $70 \%-130 \%$ ), and low C1q level $(29 \mu \mathrm{g} / \mathrm{mL}$; normal values $100-250 \mu \mathrm{g} / \mathrm{mL})$. Considering that symptoms appeared after the age of forty, the acquired form of C1-INH deficiency was suspected. Because this form is usually associated with a lymphoproliferative or autoimmune disease, investigations were performed in this direction. Protein electrophoresis showed monoclonal gammopathy characterised as immunoglobulin M.

Abdominal ultrasonography revealed splenomegaly (Figure 1) and a bone marrow biopsy was normal. No anti-C1-INH antibodies (IgG, IgM, IgA) were detected. Rupatadine (J. Uriach y Compañia., SA, Barcelona, Spain), $10 \mathrm{mg}$, 4x1 tablets/day, was administered orally, between $2^{\text {nd }}$ October and $30^{\text {th }}$ November 2018. From $1^{\text {st }}$ December 2018, the dose was decreased to $2 \mathrm{x} 1 \mathrm{tb} /$ day until $31^{\text {st }}$ January 2019.

The final diagnosis of C1-INH-AAE associated with monoclonal gammopathy of uncertain significance (MGUS) was made. The haematologist prescribed systemic corticosteroids, Dexamethasone (KRKA d.d. Novo Mesto, Slovenia) $8 \mathrm{mg} / 2 \mathrm{ml}$, one injection per day intramuscularly, between $29^{\text {th }}$ November and $10^{\text {th }}$ December 2018. Since $11^{\text {th }}$ December the treatment was changed to Prednisone (Gedeon Richter Romania SA, Targu Mures, Romania), 5mg, three tablets per day, administered orally, until $23^{\text {rd }}$ December, but the mild and moderate angioedema attacks of the limbs continued. The treatment was discontinued by $23^{\text {rd }}$ December, after 26 days of treatment, when the patient complained of adverse effects.

In January 2019, three months since her visit to the HAE Centre the patient presented at the ED with facial oedema (Figure 2), dysphagia, and dysphonia which had started 30 minutes before being admitted.

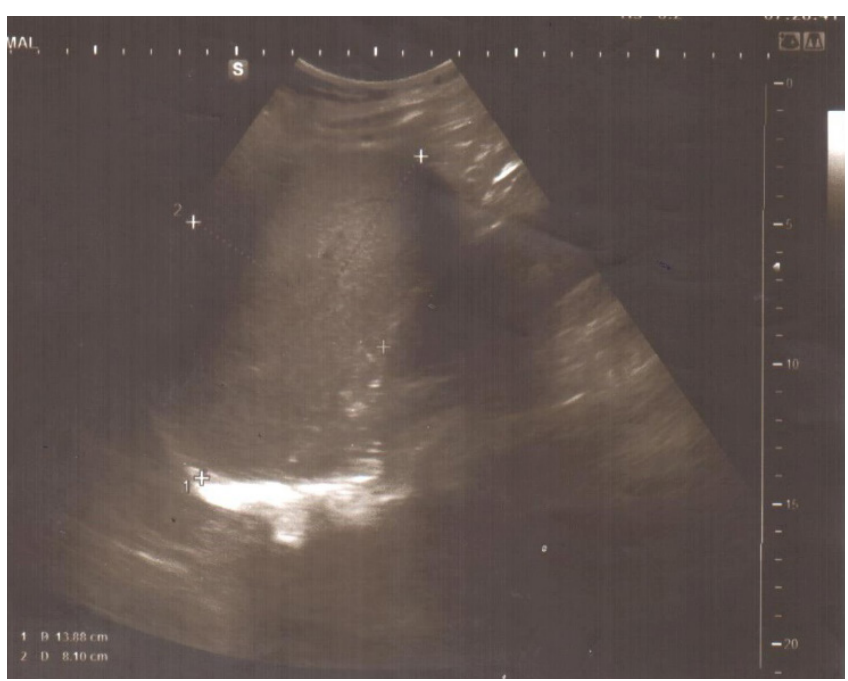

Fig. 1. Abdominal ultrasonography showing splenomegaly in a patient with hereditary angioedema and monoclonal gammopathy of uncertain significance

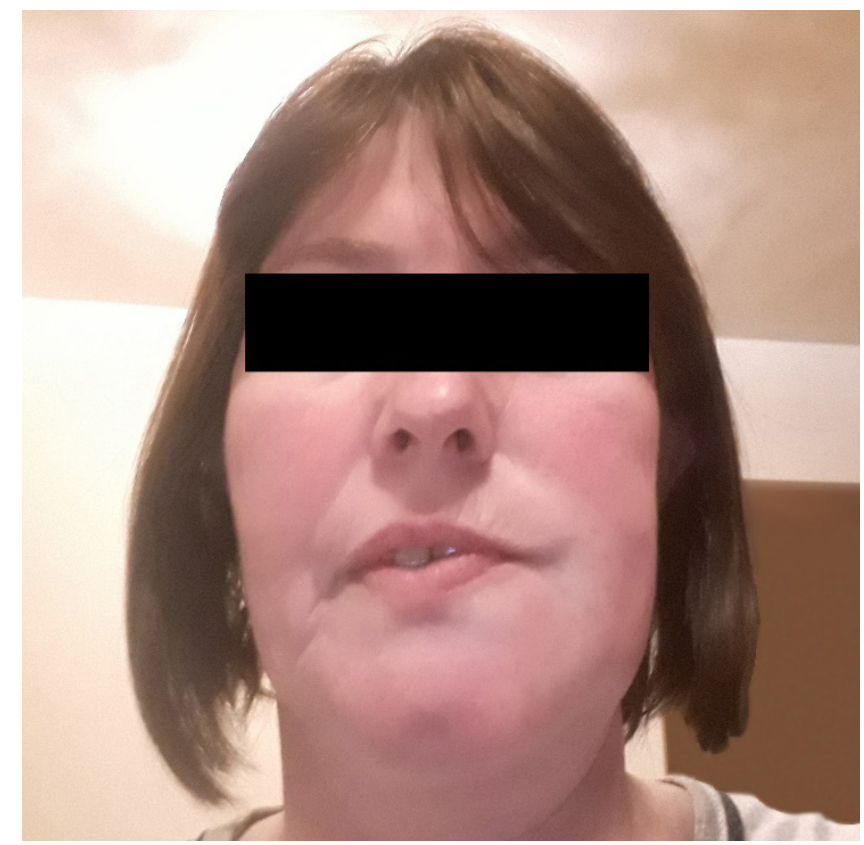

Fig. 2. Facial oedema apparent at the time of admission to the emergency department 
Laryngeal oedema was confirmed by laryngoscopy. For this attack, two vials of recombinant human C1INH replacement therapy, rhC1-INH (Conestat alfa, Pharming Group NV - Leiden, Netherlands), 2100 IU/ vial, were administered by intravenous injection on $18^{\text {th }}$ January 2019 , based on the accompanying medical letter's recommendation. All symptoms noticed at the time of admission completely subsided within six hours after the patient was given the medication. Two months later, on $15^{\text {th }}$ March 2019, she had another facial attack with dysphagia treated in the ED with the same drug. The symptom improved immediately, and she was discharged from the ED three hours after the medication had been administered. A third episode occurred two weeks following the second one, on $31^{\text {st }}$ March. The patient exhibited facial oedema with erythema, dysphagia, and upper airways swelling. Posterior pharynx and uvula oedema were visualised by laryngoscopy. Because rhC1-INH was not available at this time, this attack was treated with fresh frozen plasma (FFP) (HBP, Cluj Napoca, Romania,) 250 ml-1IU, administered by intravenous transfusion).

While the second unit of FFP was being administered, the patient experienced abdominal pain, hypotension, and nausea. Therefore, the medication was discontinued. The patient was discharged from the ED twenty hours later.

As prophylaxis against further angioedema attacks, the patient was prescribed prophylactic treatment with antifibrinolytics, Exacyl (Sanofi-Aventis Sp, Rzeszow, Poland), $500 \mathrm{mg}$, in a dose of $3 \mathrm{~g}$ per day ( $3 \times 2$ tablets per day), administered orally from $2^{\text {nd }}$ April.

No further attacks were reported at the eighteenmonth follow-up visit.

\section{DISCUSSION}

When seeing recurrent episodes of non-itching, nonpitting cutaneous or mucous membranes swelling in patients forty years of age or older, having no known relatives with angioedema, clinicians should contemplate a diagnosis of an acquired form of C1-INH deficiency. The cause of the attacks is often not apparent; the most commonly noticed triggers are emotional stressors, traumatic injuries, or infections. In this form of angioedema, any body part may be involved, including the skin, the digestive tract, and the upper airways [6]. Cutaneous swelling can manifest as non-pitting oedema of the skin lasting two to five days. Swelling of the gastrointestinal tract can result in nonspecific colicky abdominal pain, vomiting, diarrhoea, and abdominal distension. Upper airways oedema is clinically characterised by hoarseness, stridor, and dysphagia. As a life-threatening disorder which may lead to asphyxia, laryngeal or pharyngeal oedema calls for urgent medical intervention [5].

The current patient was diagnosed with $\mathrm{C} 1-\mathrm{INH}-$ AAE based on the specific clinical symptoms of recurrent episodes of swelling without urticaria, which started at the age of forty-two, in the absence of familial history. There were associated with complement deficiencies, low C1-INH activity and antigen, reduced C4 and $\mathrm{C} 1 \mathrm{q}$ level, though $\mathrm{C} 3$ levels were normal.

When C1-INH deficiency is suspected, C4 levels should be determined as a first screening test to exclude histamine-mediated oedema. This is particularly important in the ED, where a total complement measurement would not be feasible [5]. In some cases, because of an increased level of cleaved inactive C1-INH in plasma, the C1-INH antigen values fall in the normal reference range. The $\mathrm{Clq}$ level is usually far below $50 \%$ of the typical values, but a minority of patients may have normal C1q [5].

Its depletion causes the drop of C1-INH levels in AEE by the lymphoproliferative tissue or by the neutralising autoantibodies [4-6]. In approximately $70 \%$ of the patients with $\mathrm{C} 1-\mathrm{INH}-\mathrm{AEE}$, an associated malady could be identified $[6,9]$. One in two of these patients are diagnosed with a neoplastic condition, especially non-Hodgkin lymphoma, while the others with benign disease, such as MGUS. Otani and Banerji (2017) have shown that $\mathrm{C} 1$-INH-AEE is most frequently associated with indolent lymphoma and MGUS [6]. Therefore, when C1-INH-AEE diagnosis is confirmed, a thorough medical assessment should be performed to uncover any likely related conditions such as lymphoproliferative or autoimmune diseases $[6,11]$. In the present case, MGUS was diagnosed, possibly causing the over intake of the C1-INH enzyme resulting in no anti-C1INH antibodies being detected.

C1-INH-AAE may also appear, related to other conditions such as HIV infection and hepatitis B infections, vascular disease, e.g., Churg Strauss syndrome, and metabolic conditions such as xanthomatosis $[4,6]$. In our patient, there were no signs, symptoms, or laboratory results related to these disorders.

The comprehensive management of C1-INH-AAE seeks to prevent symptoms and improve the patient's 
quality of life and identify and treat any of the latent related diseases [4-6]. Considering the lack of rigorous trials conducted on patients with C1-INH-AEE, specific drugs have not yet been approved to treat this rare disease. Since the acquired form of C1-INH deficiency exhibits the same symptoms as the hereditary form, the drugs with proven efficacy in the treatment of the latter type are, by extension, prescribed in C1-INH-AEE as well [5]. In C1-INH deficiency, due to a lack of the inhibitory effects of C1-INH on FXIIa and kallikrein, the over activation of the kallikrein-kinin system induces an excessive production of bradykinin, followed by vasodilatation, vascular hyperpermeability, and eventually, swelling [2]. Therefore, this type of angioedema will respond to kallikrein-bradykinin targeted therapy but not to antihistamines, corticosteroids, or epinephrine. Acute treatment aims to reduce the duration and severity of the attack. It includes human plasma-derived C1INH concentrate (pdC1-INH) or recombinant human C1-INH concentrate (rhC1-INH), icatibant, a synthetic peptidomimetic drug acting as a specific antagonist of bradykinin B2 receptors, and ecallantide, a selective plasma kallikrein inhibitor. If these drugs are not available, FFP should be administered [4-6].

The goal of prophylactic medication is to prevent or minimise the frequency and severity of the acute episodes and include C1-INH concentrate administered every 3 to 4 days, attenuated androgens or antifibrinolytic agents [2-5].

While many angioedema attacks subside spontaneously, the risk of asphyxiation by upper airway oedema must not be played down. Early recognition of attacks is crucial and equipping the patient with printed instructions for emergency intervention is instrumental in providing proper and prompt acute treatment [6].

Patients themselves should be educated to avoid the possible triggers of the attacks, such as social stressors, trauma, exogenous oestrogens, tamoxifen, or ACEIs, and should have appropriate access to acute treatment. When necessary, they should have a prophylaxis plan $[2,4-6]$.

When no associated condition is identified initially, and because C1-INH-AAE patients are at a higher risk of developing lymphoproliferative diseases than the general population, adequate screening methods for B cell malignancies should be carried out. In most cases, treating the underlying illness in C1-INH-AAE patients results in partial or complete clinical and biochemical remission of $\operatorname{AAE}[6,12,13]$.

\section{- CONCLUSIONS}

Angioedema, especially the laryngeal swelling, is a relatively common presentation in the ED. Without treatment, the probability of asphyxiation is high. Hence it is essential to distinguish between different types of angioedema to apply the appropriate treatment as soon as possible. C1-INH-AAE is a rare disorder in which the swelling is bradykinin mediated; therefore, it will respond to kallikrein-bradykinin targeted drugs, not antihistamines, corticosteroids, or epinephrine. Because it is not easy to distinguish the different types of angioedema just on clinical symptoms, the patient must have a written treatment plan for emergency care physicians.

\section{口CONFLICT OF INTEREST}

None to declare.

\section{PATIENT CONSENT}

The patient has provided a writen consent regarding the publication of her picture.

\section{REFERENCES}

1. Bernstein JA, Moellman J. Emerging concepts in the diagnosis and treatment of patients with undifferentiated angioedema. Int J Emerg Med. 2012;5:39.

2. Bernstein JA, Cremonesi P, Hoffmann TK, Hollingsworth J.: Angioedema in the emergency department: a practical guide to differential diagnosis and management, Int J Emerg Med. 2017;10:15.

3. Lombardi C, Crivellaro M, Dama A, Senna G, Gargioni S, Passalacqua $G$. Are physicians aware of the side effects of angiotensin-converting enzyme inhibitors?: a questionnaire survey in different medical categories. Chest. 2005;128:976-9.

4. Cicardi M, Zanichelli A. Acquired angioedema. Allergy Asthma Clin Immunol. 2010;6:14.

5. Wu MA, Castelli R. The Janus faces of acquired angioedema: C1-inhibitor deficiency, lymphoproliferation and autoimmunity. Clin Chem Lab Med. 2016;54:207-14.

6. Otani IM, Banerji A. Acquired C1-inhibitor deficiency, Immunol Allergy Clin North Am. 2017; 37:497-511.

7. Bouillet-Claveyrolas L, Ponard D, Drouet C, Massot C. Clinical and biological distinctions between type I and type II acquired angioedema. Am J Med. 2003;115:420-1.

8. Dobson G, Edgar D, Trinder J. Angioedema of the tongue due to acquired $\mathrm{C} 1$ esterase inhibitor deficiency. Anaesth Intensive Care. 2003;31:99-102. 
140 - The Journal of Critical Care Medicine 2021;7(2)

9. Cicardi M, Zanichelli A. The acquired deficiency of C1inhibitor: Iymphoproliferation and angioedema. Curr Mol Med. 2010;10:354-60.

10. Cicardi M. Acquired C1 inhibitor deficiency: clinical manifestations, epidemiology, pathogenesis, and diagnosis - UpToDate. Available at: https://www.uptodate.com/ contents/acquired-c1-inhibitor-deficiency-clinicalmanifestationsepidemiology-pathogenesis-anddiagnosis?source5search_result\&search5acquiredangioedem a\&selectedTitle51w32. Accessed: 4th December, 2016.
Available online at: www.jccm.ro

11. Farkas H, Veszeli N, Kajdácsi E, Cervenak L, Varga L. "Nuts and bolts" of laboratory evaluation of angioedema. Clin Rev Allergy Immunol. 2016;51:140-51.

12. Lam DH, Levy NB, Nickerson JM, Gruenberg DA, Lansigan F. Acquired angioedema and marginal zone lymphoma. J Clin Oncol. 2012;30:e151-3.

13. Branellec A, Bouillet L, Javaud N et al. Acquired C1-inhibitor deficiency: 7 patients treated with rituximab. J Clin Immunol. 2012;32:936-41. 